Special issue of the International Conference on Computational and Experimental Science and Engineering (ICCESEN 2014)

\title{
The Measurement of Gamma Dose in Radiotherapy Unit
}

\author{
İ. $\operatorname{AKKURT}^{a}$, Ü. KARA ${ }^{b, *}$, M. YILDIZ ${ }^{c}$ AND V. KAYA ${ }^{c}$ \\ ${ }^{a}$ Suleyman Demirel University, Physics Department, Isparta, Turkey \\ ${ }^{b}$ Suleyman Demirel University, Vocational School of Healt Services, Isparta, Turkey \\ ${ }^{c}$ Suleyman Demirel University Medical Faculty Isparta, Turkey
}

\begin{abstract}
Cancer is one of the most deadly diseases posing threat for our health. The most important method for eradicating of cancer cells is photoradiotherapy. Electrons accelerated by the accelerator are converted to photons in the process of bremsstrahlung. These photons are focused on diseased cells. Photon leaving accelerator head should assure a given dose intensity in cancer cells. Measuring of beam parameters at apparatus output is essential to determine the dose. In this study, Suleyman Demirel University research and education hospital in radiation Oncology department which has located at $18 \mathrm{MeV}$ accelerator in energy gamma dose was measured.
\end{abstract}

DOI: 10.12693/APhysPolA.128.B-367

PACS: 87.56.- $\mathrm{v}, 87.55 . \mathrm{Qr}$

\section{Introduction}

Cancer is one of the main diseases to threat for human health. Besides surgery and medical treatment (chemotherapy), radiotherapy is commonly used treatment method to kill cancer cell. For this method high energy photon is required. Clinic Linear Accelerator (cLINAC) relatively lower energy than other LINAC has been used to obtain photon via bremsstrahlung processes. When accelerated electron beam impinges to the thin target bremsstrahlung occurs and photon can be created.
Those photons can be focused on cancer cell and kill cancer. In order to focus photon beam collimator materials in LINAC head generally contains heavy elements is used and the interaction of bremsstrahlung photon with the such heavy nuclei the neutron can be produced inside the treatment rooms. The production of neutron associated to gamma capture becomes significant when the energy of a linear accelerator is higher than $10 \mathrm{MeV}$. In this study gamma dose rate has been measured in radiotherapy room $[1-3]$.
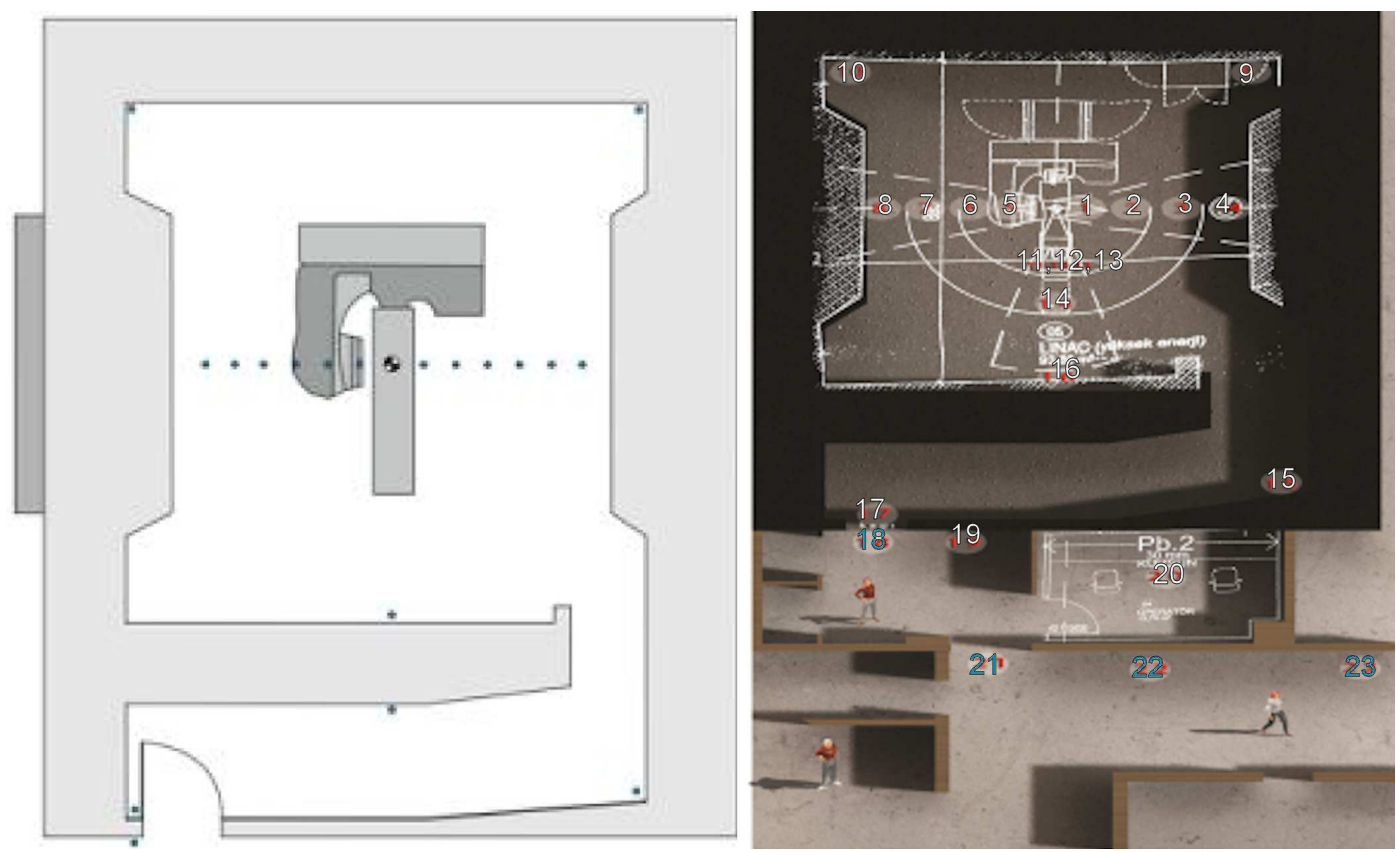

Fig. 1. 2D-3D medical linac measurements area (points 1-23).

*corresponding author; e-mail: umitkara@sdu.edu.tr 


\section{Material and method}

Radiation has existed since creation of universe and started to be used in a large of different fields nowadays. As human health is important one of the main fields of radiation utilization is medical science, especially in the diagnostic and treatment of diseases. Af-
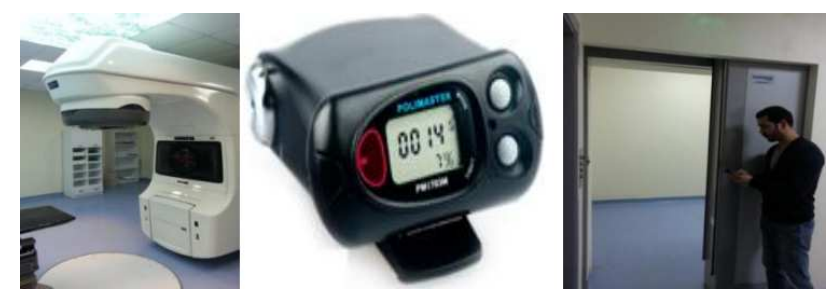

Fig. 2. Medical linac used in experiments.

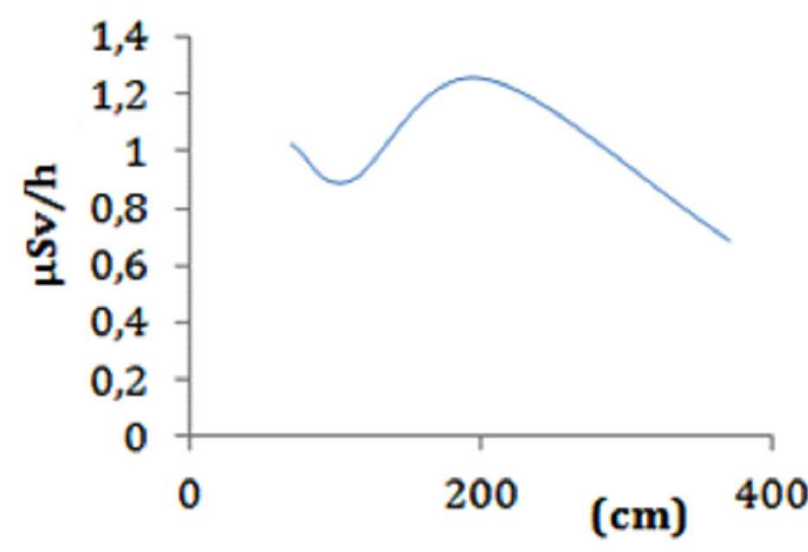

Fig. 4. Gamma dose graphics (points 1-4).

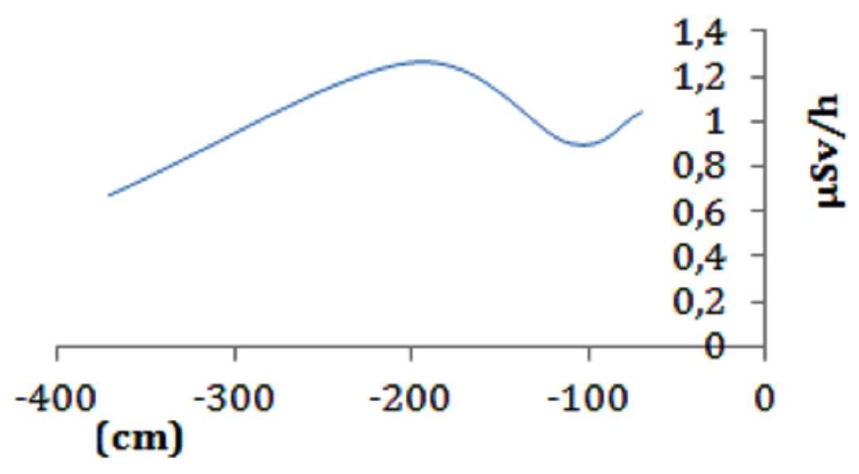

Fig. 5. Gamma dose graphics (points 5-8).

ter chemothrapy treatment photon radiotherapy is commonly used method in cancer treatment. Photon is generated from the high-energy electron accelerators (LINAC) where an electron beam can be accelerated up to about $20 \mathrm{MeV}$. In medical equipment electron beam impinges into thin sheet of metal photons are created

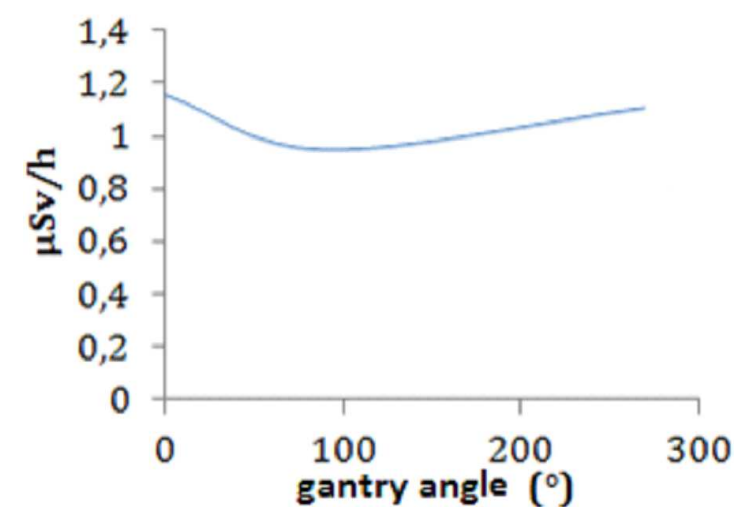

Fig. 6. Gamma dose graphics gantry angles $\left(0^{\circ}\right)$ (points 11-13).

TABLE

Measured gamma doses.

\begin{tabular}{c|c}
\hline \hline point & {$[\mu \mathrm{SV} / \mathrm{h}]$} \\
\hline 1 & 1.03 \\
2 & 0.9 \\
3 & 1.26 \\
4 & 0.69 \\
5 & 1.04 \\
6 & 0.9 \\
7 & 1.26 \\
8 & 0.67 \\
9 & 0.56 \\
10 & 0.59 \\
11 (angle of table $0^{\circ}$ ) & 1.16 \\
13 (angle of table $90^{\circ}$ ) & 0.95 \\
of table $270^{\circ}$ ) & 1.11 \\
14 & 0.91 \\
15 & 0.1 \\
16 & 0.1 \\
17 & 0.1 \\
18 & 0.1 \\
19 & 0.1 \\
20 & 0.1 \\
21 & 0.1 \\
22 & 0.1 \\
23 & 0 \\
&
\end{tabular}

via bremsstrahlung processes hospital. The photoneutrons are produced in electron linear accelerators when the bremsstrahlung energy exceeds the threshold energy of photonuclear reactions of the materials [1]. A significant number of neutrons are generated via $(\gamma, \mathrm{n})$ reaction. Those contamination comes from the target, the filter, the collimator and any places struck by high energy Xrays $([1-6])$. Besides the known effects of radiation, the effects of surgical operations are still largely unknown. Thus it is important and also difficult to measure doses of physicians and their patients during radioatherapy treatment. In this work, radiation dose due to the gamma 
contamination has been measured high energy medical linac we accelerated up to about $18 \mathrm{MeV}$ using gamma detector and calculated in radiotherapy room.

\section{Conclusion}

In this paper, we worked on Radiation Oncology Department in Suleyman Demirel Research Hospital. The gamma dose distribution depends on some parameters such as gantry angles. In this study neutron dose rate has been measured in several points (see Fig. 1) as a function of gantry angle and we measured these dose only one gantry angle is $0^{\circ}$. Those results showed that doses values within the treatment room are negligible.

In this study Clinic Linear Accelerator (cLINAC) we measured the differences in cross sections used for bremsstrahlung effect on result in different photon spectra generated in the target and impinging on the surface of the phantom. However, the effect of these differences on dose computations are negligible.

\section{Acknowledgments}

This work has been supported partly by the Suleyman Demirel University Foundation Unit (3194-D212).

\section{References}

[1] The 2007 Recommendations of the International Commission on Radiological Protection, Ann. ICRP 37, 1 (2007).

[2] European Guidance on Estimating Population Doses from Medical Ray Procedures, Radiation Protection 154, European Commission, Luxembourg 2008.

[3] Dosimetry in Diagnostic Radiology: An international code of practice, IAEA Technical Report 457, IAEA, Vienna 2007. 